\title{
A novel equation and nomogram including body weight for estimating prostate volumes in men with biopsy-proven benign prostatic hyperplasia
}

\author{
Yasukazu Nakanishi ${ }^{1}$, Hitoshi Masuda ${ }^{1}$, Satoru Kawakami ${ }^{1}$, Mizuaki Sakura ${ }^{1}$, Yasuhisa Fujii ${ }^{2}$, Kazutaka Saito ${ }^{1}$, \\ Fumitaka Koga $^{1}$, Masaya Ito ${ }^{2}$, Junji Yonese ${ }^{2}$, Iwao Fukui ${ }^{2}$ and Kazunori Kihara ${ }^{1}$
}

Anthropometric measurements, e.g., body weight (BW), body mass index (BMI), as well as serum prostate-specific antigen (PSA) and percent-free PSA (\%fPSA) have been shown to have positive correlations with total prostate volume (TPV). We developed an equation and nomogram for estimating TPV, incorporating these predictors in men with benign prostatic hyperplasia (BPH). A total of 1852 men, including 1113 at Tokyo Medical and Dental University (TMDU) Hospital as a training set and 739 at Cancer Institute Hospital (CIH) as a validation set, with PSA levels of up to $20 \mathrm{ng} \mathrm{ml}^{-1}$, who underwent extended prostate biopsy and were proved to have BPH, were enrolled in this study. We developed an equation for continuously coded TPV and a logistic regression-based nomogram for estimating a TPV greater than $40 \mathrm{ml}$. Predictive accuracy and performance characteristics were assessed using an area under the receiver operating characteristics curve (AUC) and calibration plots. The final linear regression model indicated age, PSA, \%fPSA and BW as independent predictors of continuously coded TPV. For predictions in the training set, the multiple correlation coefficient was increased from 0.38 for PSA alone to 0.60 in the final model. We developed a novel nomogram incorporating age, PSA, \%fPSA and BW for estimating TPV greater than $40 \mathrm{ml}$. External validation confirmed its predictive accuracy, with AUC value of 0.764 . Calibration plots showed good agreement between predicted probability and observed proportion. In conclusion, TPV can be easily estimated using these four independent predictors.

Asian Journal of Andrology (2012) 14, 703-707; doi:10.1038/aja.2012.31; published online 9 July 2012

Keywords: body weight; equation; nomogram; prediction; prostate-specific antigen; prostate volume

\section{INTRODUCTION}

Benign prostatic hyperplasia (BPH), defined as a diagnosis based on the occurrence of benign prostatic enlargement, in combination with lower urinary tract symptoms and bladder outlet obstruction, has been considered as a chronic and progressive disease. ${ }^{1}$ Baseline total prostate volume (TPV) has been linked to the progression of BPH (e.g., acute urinary retention and surgery for BPH)., ${ }^{2,3}$ Drugs known as $5 \alpha$-reductase inhibitors could decrease these risks and are therefore preferentially used in men with large TPVs. ${ }^{4}$ TPV estimations are important both for understanding the natural history of each BPH case and for determining the most appropriate initial treatment for each individual patient. ${ }^{2-4}$

Measurement of prostate-specific antigen (PSA) levels has revolutionized the diagnosis of prostate cancer (PCa). However, serum PSA, although highly sensitive, is organ-specific and not cancer-specific, thus resulting in difficulties in discriminating malignant from benign prostatic tissues. PSA velocity has also been reported to have limitations in the prediction of PCa. ${ }^{5}$ Even in patients with PCa, serum PSA reflects benign portion volume, as well as cancer volume, especially in lower PSA values. Therefore, serum PSA adjusted for non-cancer volume has been reported to have a positive association with pathology and biochemical recurrence. ${ }^{6}$ Of course, in men with clinical and pathological
BPH, serum PSA has been reported to show a positive correlation with TPV across different races. Therefore, serum PSA has been accepted as a proxy parameter of TPV among men with BPH. Moreover, some investigators have reported that free PSA (fPSA) and percent-fPSA (\% fPSA) outperform PSA as predictors of prostate enlargement without PCa. ${ }^{7-9}$

Previous studies have consistently shown that increased adiposity, as determined by various anthropometric measures, such as body weight (BW) ${ }^{10}$ body mass index (BMI ${ }^{10-12}$ and waist circumference, ${ }^{10,11}$ is positively associated with transrectal ultrasound- and with magnetic resonance imaging-measured TPV.

Although there are several predictive models estimating clinical prostatic enlargement based on levels of PSA or PSA derivatives, ${ }^{6-13}$ Jacobson et al..$^{13}$ recently reported that age and weight can be incorporated into models based on serum PSA levels to achieve greater accuracy when estimating TPV. However, predictive models including anthropometric measurements have never been investigated in Asia.

Based on these considerations, we developed a predictive model that incorporated age, PSA, \%fPSA and anthropometric measures to estimate TPV, and we constructed a nomogram that represents an easy and minimally invasive tool for estimating clinical prostatic enlargement.

${ }^{1}$ Department of Urology, Tokyo Medical and Dental University, Tokyo 113-8519, Japan and ${ }^{2}$ Department of Urology, Cancer Institute Hospital, Tokyo 135-8550, Japan Correspondence: Dr Y Nakanishi (yasnak.uro@tmd.ac.jp)

Received: 5 December 2011; Revised: 24 February 2012; Accepted: 1 March 2012; Published online: 9 July 2012 


\section{MATERIALS AND METHODS}

\section{Study subjects}

A total of 2042 Japanese men who were pathologically proved to have $\mathrm{BPH}$ by transrectal ultrasound-guided systematic extended biopsy protocols, including three-dimensional 26-core, 22-core and 14-core biopsies $^{14}$ at either Tokyo Medical and Dental University (TMDU) Hospital or Cancer Institute Hospital (CIH) between 2000 and 2010, were enrolled in this study. This study was approved by the institutional review board of each institution. Men who were diagnosed with PCa at repeat biopsy during the follow-up were excluded from the study. When a previous biopsy had been performed elsewhere, histological slides of the previous biopsy were reviewed by pathologists at each institution. Patients with PSA $\geqslant 20 \mathrm{ng} \mathrm{ml}^{-1}$ were excluded from the study to minimise the possibility of including patients with PCa. We also excluded patients with histories of $5 \alpha$-reductase inhibitor treatment, recurrent urinary tract infections or transurethral surgery, and those whose BW or height had not been recorded. The remaining 1852 men, consisting of 1113 patients at TMDU and 739 at $\mathrm{CIH}$, constituted the current study cohort.

\section{Baseline data information}

PSA, fPSA, urinalysis, and digital rectal examination (DRE) were evaluated. Tandem R (Hybritech, CA, USA) was used until February 2003, and thereafter Tosoh II (Tosoh, Tokyo, Japan) was used at TMDU; Tandem R was used until July 2003, and thereafter AxSYM (Abbott, IL, USA) was used at CIH for PSA evaluation. Based on transrectal ultrasound measurements using a $7.5-\mathrm{MHz}$ transducer at the time of the biopsy, we calculated TPV using the ellipsoid formula $(\pi / 6 \times$ width $\times$ height $\times$ length $)$. Anthropometric measurements, including BW, height, BMI and body surface area (BSA), were also determined in all of the men. BMI was calculated as the weight divided by the square of the height. BSA was calculated as $\mathrm{BW}^{0.425} \times$ height $^{0.72} \times 0.007184{ }^{15}$

We used only the latest biopsy data for each patient. Because of skewed distributions, PSA, \% fPSA and TPV were log-transformed in all of the analyses. Using data from the TMDU cohort as a training set, we performed least-squares regression to estimate TPV based on PSA alone.

\section{Creation of the equation for predicting prostate volume}

To determine the significance of the various factors-age, PSA, \%fPSA, BW, height, BMI, BSA and all two-way interactions-a multivariate backward stepwise regression analysis was used. Starting with all of the terms in the model, the least significant terms were sequentially removed until all of the remaining terms were statistically significant. Then, the equation to estimate TPV was derived. To identify the best model, Fvalues were set up with $<2.000$ to remove and $\geqslant 2.000$ to enter.

The Akaike information criterion (AIC) was used to select the best model for estimating TPV. The AIC is defined as follows: AIC $=-2 \times$ maximum log likelihood $+2 \times$ number of parameters in model. ${ }^{16}$ The formula with the smallest AIC indicated the best model.

The equation derived based on the data from the TMDU training cohort was used to estimate TPV in the $\mathrm{CIH}$ validation cohort. Linear regression $R$ values from the training set were compared with the $R$ values for prediction in the validation set.

\section{Creation of the nomogram for predicting clinical prostatic enlargement $(\mathrm{TPV}>\mathbf{4 0} \mathrm{ml})$}

We developed a nomogram for predicting TPV greater than $40 \mathrm{ml}$ using the data from 1113 men treated at TMDU (training set), and we validated the nomogram using the data from 739 men treated at $\mathrm{CIH}$ (validation set). The main advantage of the nomogram instrument is that clinicians can assess BPH risk on an individual basis and make management decisions. This cut-off point has been used in previous studies, not only for Western populations ${ }^{17,18}$ but also for Asian populations. ${ }^{7}$ Using the TMDU data set, significant and independent predictors of TPVs greater than $40 \mathrm{ml}$ were identified by univariate and multivariate analyses using a backward stepwise logistic regression. Incorporating all of the significant predictors, a logistic regression-based nomogram was developed. The predictive accuracy of the nomogram was quantified with the area under the receiver operating characteristics curve (AUC). Performance characteristics were examined using calibration plots. The extent of overestimation or underestimation was explored graphically using local regression nonparametric smoothing lines.

\section{Data analysis}

All of the analyses were performed using JMP software, version 8.0 (SAS Institute, Cary, NC, USA), and S-PLUS software, version 8 (TIBCO Software, Palo Alto, CA, USA). The difference between AUC values assessed using ROCKIT 1.1B2 software (Chicago University, Chicago, IL, USA). All calculated $P$ values were two-sided, and $P<0.05$ was considered statistically significant.

\section{RESULTS}

The baseline characteristics of the training and validation sets are presented in Table 1. The training cohort had lower PSA $(P=0.0002)$, BW $(P=0.0047)$, height $(P=0.032)$, BMI $(P=0.036)$ and BSA $(P=0.0026)$ than the validation cohort. There were no significant differences in $\%$ fPSA or TPV between the two cohorts.

As shown in Table 2, a multivariate backward stepwise regression analysis, based on the data from the training set, identified age $(P<$ $0.0001)$, PSA $(P<0.0001)$, \%fPSA $(P<0.0001)$ and BW $(P<0.0001)$ as independent predictors of TPV.

Based on the above result, we developed the equation estimating TPV as follows: $\log (\mathrm{TPV})=0.3889+0.3453 \times \log (\mathrm{PSA})+0.3460 \times$ $\log (\% \mathrm{fPSA})+0.0040 \times$ age $+0.0037 \times \mathrm{BW}$ (final model). The multiple correlation coefficient $(R)$ of the equation was 0.60 . When estimating TPV by PSA only, $R$ was 0.38 .

The prediction $R$ value applied to the validation set in the final model was 0.57 .

The formula incorporating PSA, \%fPSA, age and BW predicted TPV most accurately; this was followed by the formula incorporating PSA, \%fPSA and age; the formula incorporating PSA and \%fPSA; and the formula incorporating PSA alone, yielding AIC values of 9436.5, 9480.6, 9492.3 and 9720.5, respectively.

Age $(P=0.0002)$, PSA $(P<0.0001)$, \%fPA $(P<0.0001)$ and BW $(P<0.0001)$ were identified as statistically significant and independent predictors for the TPV grater than $40 \mathrm{ml}$ based on the training set data (Table 3). Based on the training set data, we developed a logistic regression-based nomogram (Figure 1) incorporating age, PSA, $\%$ fPSA and BW with AUC value of 0.772 . When predicting TPV greater than $40 \mathrm{ml}$ by PSA only, the AUC value was 0.657 . The AUC values for the final model were significantly higher than the model incorporating PSA only.

External validation confirmed the predictive accuracy of the nomogram, which yielded AUC values of 0.764 . The calibration plots of the nomogram indicated good agreement between the predicted probability and observed proportion of TPV (Figure 2).

\section{DISCUSSION}

In this study, using TMDU cohort (training set), we developed an equation for estimating TPV and a nomogram for predicting clinically 
Table 1 Baseline characteristics of the patients at each institution ${ }^{\mathrm{a}}$

\begin{tabular}{lccc}
\hline Variable & TMDU training set $(\mathrm{n}=1113)$ & ClH validation set $(n=739)$ & $\mathrm{P}$ value \\
\hline Age (year) & $66(61-71)$ & $65(60-70)$ & $<0.0001$ \\
PSA (ng ml ${ }^{-1}$ ) & $6.3(4.6-8.8)$ & $6.8(5.2-9.7)$ & 0.0002 \\
\%fSA (\%) & $18.7(13.9-24.4)$ & $18.8(13.8-24.2)$ & 0.52 \\
BW (kg) & $64.0(58.3-70.3)$ & $65.2(59.6-71.0)$ & 0.0047 \\
Height (cm) & $165.0(161.0-169.4)$ & $166.2(162.1-170.0)$ & 0.032 \\
BMl & $23.5(21.7-25.3)$ & $23.6(22.1-25.4)$ & 0.036 \\
BSA (m $\left.{ }^{2}\right)$ & $1.66(1.57-1.75)$ & $1.68(1.60-1.77)$ & 0.0026 \\
TPV (ml) & $40.35(30.4-53.2)$ & $38.7(30.2-51.9)$ & 0.27 \\
\hline
\end{tabular}

Abbreviations: BMI, body mass index; BSA, body surface area; BW, body weight; $\mathrm{ClH}$, Cancer Institute Hospital; PSA, prostate-specific antigen; TMDU, Tokyo Medical and Dental University; TPV, total prostate volume; \%fPSA, percent-free PSA.

${ }^{a}$ Continuous variables are expressed as median values (inter-quartile ranges).

significant TPV $(>40 \mathrm{ml})$ in Japanese patients with biopsy-proven $\mathrm{BPH}$. Actually, the addition of \% fPSA, age and BW to serum PSA increased the correlation coefficient with TPV from 0.38 to 0.60 . The AUC values of the nomogram from 0.657 to $0.772(P<0.0001)$ The above results were externally validated with good agreements using $\mathrm{CIH}$ cohort (validation set). As far as we know, this is the first study to introduce a nomogram as a tool with which to predict clinically significant TPV throughout the world. In the current study, we confirmed the significant correlation between PSA and TPV in patients with biopsy-proven BPH. Serum PSA has been accepted as a proxy parameter for TPV in different populations with similar results. ${ }^{18-23}$ The serum PSA-TPV relationship curve in the current study was somewhat similar to those in previous studies in Asian populations. ${ }^{22,23}$ Therefore, we believe that our data can be considered representative for Asian populations.

It has been shown that a lower \% fPSA enhances the detection of PCa in patients with PSA levels between 4 and $10 \mathrm{ng} \mathrm{ml}^{-1} .{ }^{24}$ In contrast, in our BPH cohort, \%fPSA was positively associated with TPV, and the use of both \% fPSA and PSA further enhanced the predictive accuracy of TPV compared to the use of PSA only. Ornstein et al. ${ }^{8}$ reported that among 70 men with biopsy-proven BPH, \%fPSA, but not PSA, was significantly correlated with TPV in patients with PSA levels ranging from 2.6 to $9.9 \mathrm{ng} \mathrm{ml}^{-1}$, indicating a predictive role of fPSA for TPV. Mao et al. ${ }^{7}$ reported that fPSA was a more accurate predictor of TPV than PSA in 286 Chinese men with biopsy-proven BPH. In the current study, the correlation coefficient of the formula incorporating PSA and $\%$ fPSA with TPV $(R=0.56)$ was equal to that of the formula using only fPSA with TPV $(R=0.56)$. However, fPSA seems to provide additional information that helps to discriminate between benign and malignant

Table 2 Univariate and multivariate backward stepwise regression analyses to determine the predictors of continuously coded TPV and $F$ value

\begin{tabular}{lcccc}
\hline \multirow{2}{*}{ Variable } & Univariate & & Multivariate & \\
\cline { 2 - 3 } & P value & & P value & F value \\
\hline Age (year) & $<0.0001$ & & $<0.0001$ & \\
PSA (ng ml & & & & \\
\%fPSA (\%) & $<0.0001$ & & $<0.0001$ & 25.6 \\
BW (kg) & $<0.0001$ & & $<0.0001$ & 210.8 \\
Height (cm) & 0.0009 & & $<0.0001$ & 52.3 \\
BMI & 0.0383 & & 0.45 & - \\
BSA (m $\left.{ }^{2}\right)$ & $<0.0001$ & & 0.47 & - \\
\hline
\end{tabular}

Abbreviations: BMI, body mass index; BSA, body surface area; BW, body weight; PSA, prostate-specific antigen; TPV, total prostate volume; \%fPSA, percent-free PSA; - , no value. disease in daily practice. Therefore, for the prediction of TPV, a combination of PSA and \%fPSA seems to be more practical than fPSA alone.

We also investigated the correlations between anthropometric measurements and TPV. Previous studies have consistently shown that increased adiposity, as determined by various anthropometric measurements, such as BW, ${ }^{10} \mathrm{BMI}^{10-12}$ and waist circumference, ${ }^{10,11}$ are positively associated with TPV. We confirmed that among BW, height, BMI and BSA, BW was the most powerful independent predictor of TPV after adjustment for PSA, \%fPSA and age using a backward stepwise regression model and that no combination of anthropometric measurements was superior to BW alone. Based on the aforementioned results, we established an equation consisting of PSA, \% fPSA, age and BW. Jacobson et al. ${ }^{13}$ already reported an equation incorporating PSA, age and BW for predicting TPV in Western populations. It seems practical to be aware of the usefulness of clinically convenient data, such as age and $\mathrm{BW}$, to manage $\mathrm{BPH}$, irrespective of race.

Some researchers reported mechanisms between obesity and TPV. One explanation is systemic inflammation. Obesity and metabolic syndrome are associated with systemic inflammation and oxidative stress. BPH in surgical specimens is associated with inflammation, and the extent and severity of inflammation correspond to the amount of prostate enlargement. Thus, it is possible that systemic inflammatory mediators and oxidative stress related to obesity promote prostate growth. Wu et al. ${ }^{25}$ reported that prostate hyperplasia caused by an immune inflammatory process might contribute to $\mathrm{BPH}$ progression. Another possible explanation is that sex steroid hormone levels change due to obesity. Increased adiposity promotes increased aromatisation of circulating testosterone into oestrogen; such shifts in the balance between testosterone and oestrogen in prostate tissue may contribute to $\mathrm{BPH}$ pathogenesis. ${ }^{26}$

Recently, obese men have been reported to have larger plasma volumes, which could decrease their serum PSA levels. This effect, known as haemodilution, could lead to lower PSA levels in obese men. ${ }^{27,28}$ Thus, it is likely that if serum PSA is the same between obese and non-obese men, then the prostatic tissue in the former group will release more PSA protein than that in the latter group. Thus, the relationship between serum PSA and TPV must be affected by obesity. Obesity is apparently more common in Caucasian men than in Japanese men. ${ }^{29}$ Therefore, racial differences in obesity status might explain the higher levels of serum PSA per unit of TPV in Japanese vs. Caucasian American men in addition to the different cellular composition of BPH, with a higher glandular component and a lower stromal component in Japanese men. The inclusion of anthropometric parameters in the estimation of TPV might be useful for estimating TPV, especially in groups with a wide range of body size or among different ethnicities. 
Table 3 Univariate and multivariate backward stepwise logistic regression analyses for the estimation of TPV $>40 \mathrm{ml}$

\begin{tabular}{|c|c|c|c|c|c|}
\hline \multirow{3}{*}{ Variable } & \multicolumn{5}{|c|}{$T P V>40 \mathrm{ml}$} \\
\hline & \multicolumn{2}{|c|}{ Univariate } & \multicolumn{3}{|c|}{ Multivariate } \\
\hline & $\mathrm{P}$ value & predictive accuracy & OR $(95 \% \mathrm{Cl})$ & $\mathrm{P}$ value & predictive accuracy \\
\hline PSA $\left(n g \mathrm{ml}^{-1}\right)$ & $<0.0001$ & 0.657 & $0.023(0.011-0.047)$ & $<0.0001$ & \\
\hline$\%$ PPSA (\%) & $<0.0001$ & 0.681 & $0.009(0.0042-0.023)$ & $<0.0001$ & 0.772 \\
\hline BW (kg) & 0.0056 & 0.544 & $0.96(0.94-0.97)$ & $<0.0001$ & \\
\hline $\operatorname{BSA}\left(\mathrm{m}^{2}\right)$ & 0.1664 & - & - & - & - \\
\hline
\end{tabular}

Abbreviations: BMI, body mass index; BSA, body surface area; BW, body weight; $\mathrm{Cl}$, confidence interval; OR, odds ratio; PSA, prostate-specific antigen; TPV, total prostate volume; \%fPSA, percent-free PSA; -, no value.

Because the degree of prostatic enlargement that results in $\mathrm{BPH}$ progression-defined as a relatively high International Prostate Symptom Score, acute urinary retention, or the need for surgeryvaries from study to study, we evaluated the ability of a new formula incorporating age, PSA, \% fPSA and BW to predict prostatic enlargement at a relatively wide range of levels, namely, a TPV $>40 \mathrm{ml}$. For this range of prostate sizes, receiver operating characteristics analysis confirmed that the new formula outperformed PSA alone in its ability to predict clinically significant prostate enlargement. Based on the above results, we constructed a new nomogram for predicting $\mathrm{BPH}$. There has been no previous establishment of nomograms that estimate prostatic enlargement associated with $\mathrm{BPH}$.

This study was based on cross-sectional findings; further longitudinal studies should be performed to clarify the impact of anthropometric measurements on TPV.

To our knowledge, our current cohort is one of the largest Asian ones on the association of PSA or PSA derivatives with prostate volume in

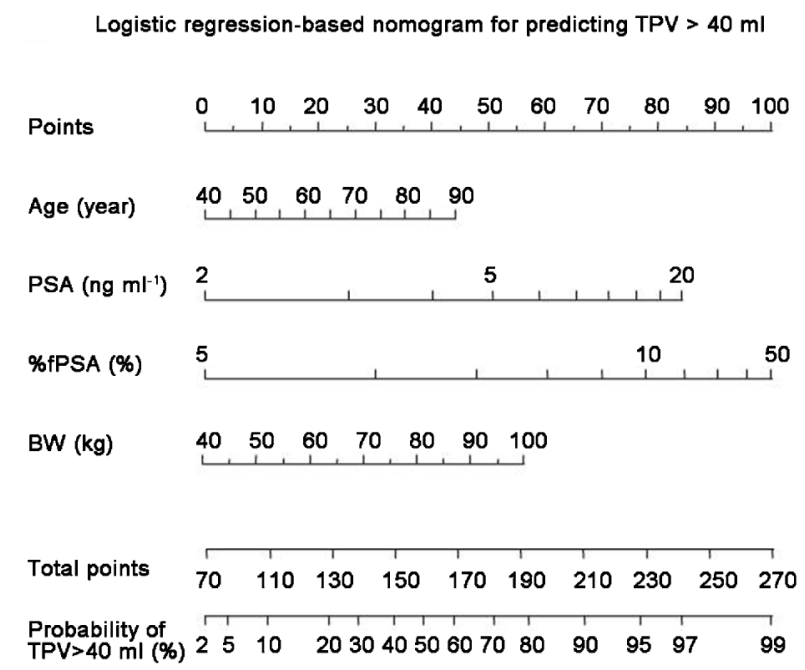

Figure 1 Logistic regression-based nomogram predicting TPV $>40 \mathrm{ml}$. The probability that prostate volume is greater than these cut-off points $(P)$ is given by the formula $P=1 /(1+\exp (Y))$, where $Y=-14.150+3.837 \times \log (P S A)+4.559 \times$ $\log (\% \mathrm{fPSA})+0.040 \times$ age $+0.043 \times \mathrm{BW}$. To obtain the predicted probability of prostate volume, locate the patient values on each axis. Draw a vertical line upward to the 'Points' axis to determine the points of the variable. Add together all of the points for all variables, and locate the sum on the 'Total points' axis. Draw a vertical line down to the 'Probability of TPV $>40 \mathrm{ml}$ axis to find the patient's probability. BW, body weight; PSA, prostate-specific antigen; TPV, total prostate volume; \%fPSA, percent-free PSA. biopsy-proven BPH. Our analysis was limited to biopsy-proven BPH cases. The median PSA $\left(6.3 \mathrm{ng} \mathrm{ml}^{-1}\right)$ in our cohort was higher than normal, although the possibility of concomitant PCa seems to be decreased by extended prostate biopsy as much as possible. Therefore, we acknowledge that this is a limitation of the applicability of our data to the general Japanese population with clinically diagnosed $\mathrm{BPH}$. However, patients with elevated PSA and negative extended biopsies, irrespective of lower urinary tract symptoms, can be expected to develop bladder outlet obstruction. ${ }^{30}$ Thus, biopsy-proven BPH cohorts, including ours, should be checked more closely in functional studies.

\section{CONCLUSIONS}

We developed a novel prostate volume-estimating equation and a nomogram incorporating age, PSA, \%fPSA and BW based on a cohort with extended biopsy-proven BPH. TPV can be easily estimated using these four independent predictors.

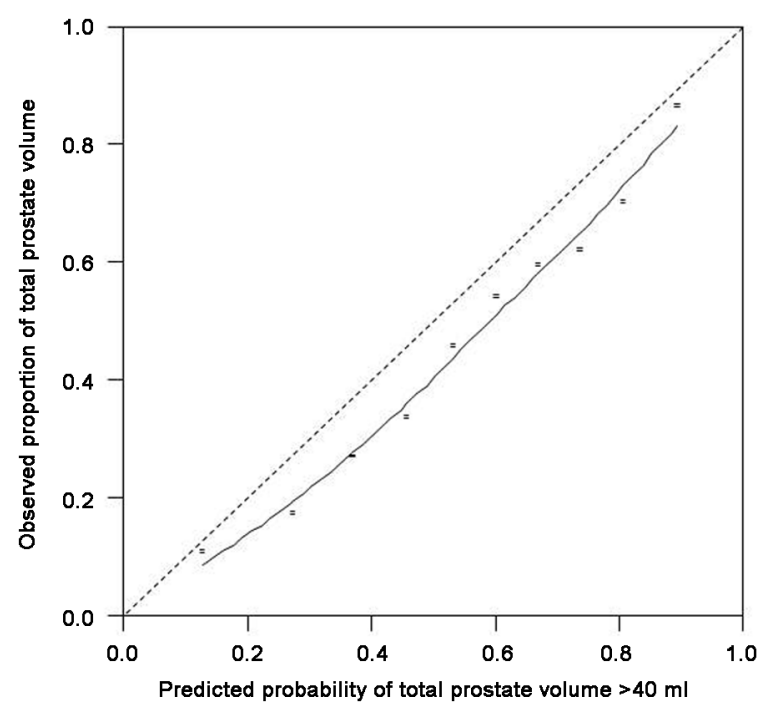

Figure 2 Calibration plots with non-parametric smoothing lines. X-axis represents the nomogram-predicted probability of TPV, and y-axis represents the observed proportion of TPV. Perfect predictions correspond to the $45^{\circ}$ line. Points established below the $45^{\circ}$ line correspond to nomogram over-prediction, whereas points situated above the $45^{\circ}$ line correspond to nomogram underprediction. TPV, total prostate volume. 


\section{AUTHOR CONTRIBUTIONS}

YN designed the study, performed the statistical analysis and wrote the paper; HM made substantial contributions to the conception and design of the study and to the interpretation of the data; SK, MS, YF, KS, FK, MI, JY and IF participated in critically revising the paper for key intellectual content; and KK participated in designing the study, interpreting the data and critically revising the paper for key intellectual content. All of the authors read and approved the final manuscript.

\section{COMPETING FINANCIAL INTERESTS}

The authors declare that they do not have any competing financial interests.

1 Emberton M, Andriole GL, de la Rosette J, Djavan B, Hoefner K et al. Benign prostatic hyperplasia: a progressive disease of aging men. Urology 2003: 61: 267-73.

2 Lieber MM, Jacobsen SJ, Roberts RO, Rhodes T, Girman CJ. Prostate volume and prostate-specific antigen in the absence of prostate cancer: a review of the relationship and prediction of long-term outcomes. Prostate 2001; 49: 208-12.

3 Marberger MJ, Andersen JT, Nickel JC, Malice MP, Gabriel M et al. Prostate volume and serum prostate-specific antigen as predictors of acute urinary retention. Combined experience from three large multinational placebo-controlled trials. Eur Urol 2000; 38: 563-8.

4 McConnell JD, Bruskewitz R, Walsh P, Andriole G, Lieber M et al. The effect of finasteride on the risk of acute urinary retention and the need for surgical treatment among men with benign prostatic hyperplasia. Finasteride Long-Term Efficacy and Safety Study Group. N Engl J Med 1998; 338: 557-63.

5 Borofsky MS, Makarov DV. Prostate-specific antigen (PSA) velocity: a test of controversial benefit in the era of increased prostate cancer screening. Asian J Androl 2011; 13: 614-5.

$6 \mathrm{Ku} \mathrm{JH}$, Moon KC, Cho SY, Kwak C, Kim HH. Serum prostate-specific antigen value adjusted for non-cancerous prostate tissue volume in patients undergoing radical prostatectomy: a new predictor of biochemical recurrence in localized or locally advanced prostate cancer. Asian J Androl 2011; 13: 248-53.

7 Mao Q, Zheng X, Jia X, Wang Y, Qin J et al. Relationships between total/free prostatespecific antigen and prostate volume in Chinese men with biopsy-proven benign prostatic hyperplasia. Int Urol Nephrol 2009; 41: 761-6.

8 Ornstein DK, Smith DS, Humphrey PA, Catalona WJ. The effect of prostate volume, age, total prostate specific antigen level and acute inflammation on the percentage of free serum prostate specific antigen levels in men without clinically detectable prostate cancer. J Urol 1998; 159: 1234-7.

9 Steuber T, Niemela P, Haese A, Pettersson K, Erbersdobler A et al. Association of freeprostate specific antigen subfractions and human glandular kallikrein 2 with volume of benign and malignant prostatic tissue. Prostate 2005; 63: 13-8.

10 Parsons JK, Carter HB, Partin AW, Windham BG, Metter EJ et al. Metabolic factors associated with benign prostatic hyperplasia. J Clin Endocrinol Metab 2006; 91: 2562-8.

11 Lee S, Min HG, Choi SH, Kim YJ, Oh SW et al. Central obesity as a risk factor for prostatic hyperplasia. Obesity 2006; 14: 172-9.
12 Sarma AV, Jaffe CA, Schottenfeld D, Dunn R, Montie JE et al. Insulin-like growth factor-1, insulin-like growth factor binding protein-3, and body mass index: clinical correlates of prostate volume among Black men. Urology 2002; 59: 362-7.

13 Jacobson DJ, St Sauver JL, Parker AS, McGree ME, Sarma AV et al. Estimation of prostate size in community-dwelling men. Urology 2011; 77: 422-6.

14 Kawakami S, Numao N, Okubo Y, Koga F, Yamamoto S et al. Development, validation, and head-to-head comparison of logistic regression-based nomograms and artificial neural network models predicting prostate cancer on initial extended biopsy. Eur Urol 2008; 54: 601-11.

15 Du bois D, Du bois EF. A formula to estimate the approximate surface area if height and weight be known. Arch Intern Med 1916; 17: 863-71.

16 Akaike $\mathrm{H}$. A new look at the statistical model identification. IEEE Trans Autom Control 1974; 19: 716-23.

17 Roehrborn CG, McConnell JD, Lieber M, Kaplan S, Geller J et al. Serum prostate-specific antigen concentration is a powerful predictor of acute urinary retention and need for surgery in men with clinical benign prostatic hyperplasia. Urology 1999; 53: 473-80.

18 Bohnen AM, Groeneveld FP, Bosch JL. Serum prostate-specific antigen as a predictor of prostate volume in the community: the Krimpen study. Eur Urol 2007; 51: 1645-53.

19 Roehrborn CG, Boyle P, Gould AL, Waldstreicher J. Serum prostate-specific antigen as a predictor of prostate volume in men with benign prostatic hyperplasia. Urology 1999; 53: 581-9.

20 Lee SE, Chung JS, Han BK, Moon KH, Hwang SI et al. Relationship of prostatespecific antigen and prostate volume in Korean men with biopsy-proven benign prostatic hyperplasia. Urology 2008; 71: 395-8.

21 Hochberg DA, Armenakas NA, Fracchia JA. Relationship of prostate-specific antigen and prostate volume in patients with biopsy proven benign prostatic hyperplasia. Prostate 2000; 45: 315-9.

22 Gupta A, Aragaki C, Gotoh M, Masumori N, Ohshima S et al. Relationship between prostate specific antigen and indexes of prostate volume in Japanese men. J Urol 2005; 173: 503-6.

23 Chang YL, Lin AT, Chen KK, Chang YH, Wu HH et al. Correlation between serum prostate specific antigen and prostate volume in Taiwanese men with biopsy proven benign prostatic hyperplasia. J Urol 2006; 176: 196-9.

24 Mettlin C, Chesley AE, Murphy GP, Bartsch G, Toi A et al. Association of free PSA percent, total PSA, age, and gland volume in the detection of prostate cancer. Prostate 1999; 39: 153-8.

25 Wu ZL, Yuan Y, Geng H, Xia SJ. Influence of immune inflammation on androgen receptor expression in benign prostatic hyperplasia tissue. Asian J Androl 2012; 14: 316-9.

26 Ho CK, Nanda J, Chapman KE, Habib FK. Oestrogen and benign prostatic hyperplasia: effects on stromal cell proliferation and local formation from androgen. J Endocrinol 2008; 197: 483-91.

27 Banez LL, Hamilton RJ, Partin AW, Vollmer RT, Sun L et al. Obesity-related plasma hemodilution and PSA concentration among men with prostate cancer. JAMA 2007; 298: 2275-80.

28 Grubb RL 3rd, Black A, Izmirlian G, Hickey TP, Pinsky PF et al. Serum prostatespecific antigen hemodilution among obese men undergoing screening in the Prostate, Lung, Colorectal, and Ovarian Cancer Screening Trial. Cancer Epidemiol Biomarkers Prev 2009; 18: 748-51.

29 Ando R, Nagaya T, Hashimoto Y, Suzuki S, Itoh Y et al. Inverse relationship between obesity and serum prostate-specific antigen level in healthy Japanese men: a hospitalbased cross-sectional survey, 2004-2006. Urology 2008; 72: 561-5.

30 van Renterghem K, van Koeveringe G, Achten R, van Kerrebroeck P. A new algorithm in patients with elevated and/or rising prostate-specific antigen level, minor lower urinary tract symptoms, and negative multisite prostate biopsies. Int Urol Nephrol 2010; 42: 29-38. 